CARPATHIAN J. MATH.

Volume 37 (2021), No. 2,

Pages 287 - 294
Online version at https : //www . carpathian. cunbm . utcluj. ro/

Print Edition: ISSN 1584 - 2851; Online Edition: ISSN 1843 - 4401

DOI: https://doi.org/10.37193/CJM.2021.02.14

Dedicated to Prof. Ioan A. Rus on the occasion of his $85^{\text {th }}$ anniversary

\title{
Existence of fixed points of weak enriched nonexpansive mappings in Banach spaces
}

\author{
Suthep SuAntai, DAWAn Chumpungam and PANitARn SARnMETA
}

\begin{abstract}
In this work, we introduce and study a new class of weak enriched nonexpasive mappings which is a generalization of enriched nonexpansive mappings provided by Berinde [Approximating fixed points of enriched nonexpansive mappings by Krasnoselskij iteration in Hilbert spaces, Carpathian J. Math., 35 (2019), No. 3, 293-304]. This class of mappings generalizes several important classes of nonlinear mappings. We prove some fixed point theorems regarding this kind of mappings which extend some important results in [Berinde, V., Approximating fixed points of enriched nonexpansive mappings by Krasnoselskij iteration in Hilbert spaces, Carpathian J. Math., 35 (2019), No. 3, 293-304]. Moreover, some examples, to ensure the existence of these mappings and support our main theorems, are also given.
\end{abstract}

\section{INTRODUCTION AND PRELIMINARIES}

Fixed point theory is one of the most important field of research in mathematics. Many real world problems in physics, engineering and economics can be formulated as fixed point problems which in turn can be solved with various methods in fixed point theory.

Throughout this section, unless otherwise stated, we denote $X$ to be a metric space, $C$ a nonempty subset of $X$, and $F i x(T)$ a set of all fixed points of $T$.

A mapping $T: C \rightarrow C$ is said to be a contraction if

$$
d(T x, T y) \leq c d(x, y),
$$

for some $c \in[0,1)$ and for all $x, y \in C$. The famous Banach contraction mapping principle states that in a complete metric space, any contraction has a unique fixed point which can be approximated by Picard iteration.

Over the past decades, many mathematicians have investigated important classes of contractive-like mappings.

In 1968, Kannan [17] introduced a Kannan mapping $T: C \rightarrow C$ which satisfies

$$
d(T x, T y) \leq c[d(x, T x)+d(y, T y)]
$$

for some $c \in\left[0, \frac{1}{2}\right)$ and for all $x, y \in C$. The Kannan fixed point theorem ensures that in a complete metric space, any Kannan mapping has a unique fixed point which can be approximated by Picard iteration.

In 1996, Osilike [19] considered the class of mappings $T: C \rightarrow C$ satisfying

$$
d(T x, T y) \leq c d(x, y)+L d(x, T x),
$$

for some $L \geq 0, c \in[0,1)$ and for all $x, y \in C$. He provided the T-stability of various iterations namely Mann, Ishikawa and Kirk iteration. However, these mappings do not have a fixed point, in general.

Received: 31.01.2021. In revised form: 17.05.2021. Accepted: 24.05.2021

2010 Mathematics Subject Classification. 47H09, 47H10.

Key words and phrases. enriched nonexpansive mapping, existence theorem, fixed point theory .

Corresponding author: P. Sarnmeta; panitarn_s@cmu.ac.th 

by

In 2004, Berinde [2] defined a weak contraction (also called almost contraction) $T: C \rightarrow C$

$$
d(T x, T y) \leq c d(x, y)+L(y, T x)
$$

for some $L \geq 0, c \in(0,1)$ and for all $x, y \in C$. Existence as well as existence and uniqueness results were established in [2] for almost contractions.

In 2008, Qing and Rhoades [20] studied a quasi $(L, m)$-contrative mapping defined as follows: $T: C \rightarrow C$ such that

$$
d(T x, p) \leq c d(x, p)+L d(x, T x),
$$

for some $L \geq 0, c \in[0,1)$ and for all $x \in C, p \in F i x(T)$. They proved the T-stability of Picard iteration for this kind of mappings.

Another important class of mappings, which have been introduced and studied intensely, is that of demicontractive mappings which are defined as follows: a mapping $T$ : $C \rightarrow C$, where $C$ is a nonempty subset of a Hilbert space $\mathrm{H}$, is demicontrative if $F i x(T) \neq$ $\emptyset$, and

$$
\|T x-p\|^{2} \leq\|x-p\|^{2}+k\|x-T x\|^{2},
$$

for some $k \geq 0$ and for all $x \in C, p \in F i x(T)$. Some mild conditions such as $I-T$ is demiclosed at zero and $k<1$, ensure that Mann iteration converges weakly to a fixed point of a demicontractive mapping $T$ under some suitable control sequences, for more information see $[10,13,14,16]$ and the references therein.

In 2015 Măruşter and Rus [18] studied a subclass of demicontrative mappings called strongly demicontractive mappings satisfying the following:

$$
\|T x-p\|^{2} \leq c\|x-p\|^{2}+k\|x-T x\|^{2},
$$

for some $k \geq 0, c \in[0,1)$ and for all $x \in C, p \in F i x(T)$. They proved the relation between strongly demicontractive and quasi $(L, m)$-contractive mappings as well as Kannan type mappings.

In the particular case $c=1$ in (1.1), we obtain the important class of nonexpansive mappings as follows.

A mapping $T: C \rightarrow X$ is said to be nonexpansive if

$$
\|T x-T y\| \leq\|x-y\|, \quad \text { for all } x, y \in X .
$$

However, the existence of a fixed point of a nonexpansive mapping in a complete metric space might not be ensured, in general. For example, $T x=x+1, x \in \mathbb{R}$ is obviously a nonexpansive mapping but is fixed point free.

In 1955, Krasnoselskij [17] established the following theorem which ensures the existence of a fixed point of a nonexpansive mapping.

Theorem 1.1. Let $C$ be a closed subset of a uniformly convex Banach space $X$. If T :C $\rightarrow C$ is nonexpansive and $T(C)$ is compact, then a sequence defined by $x_{n+1}=\frac{1}{2} x_{n}+\frac{1}{2} T x_{n}$ converges to a fixed point of $T$.

In 1965, Browder [5] proved the following theorem:

Theorem 1.2. Let $X$ be a uniformly convex Banach space, $T$ a nonexpansive mapping of the bounded closed convex subset $C$ of $X$ into $C$. Then $T$ has a fixed point in $C$.

More works regarding both contractive and nonexpansive mappings can be found in $[6,7,8,11,12,21]$ and the references therein.

In 2019, Berinde [3] introduced a new class of enriched nonexpansive mappings which is a generalization of that of nonexpansive mappings. It is defined as follows: 
Let $C$ be a nonempty subset of a normed space $X$. A mapping $T: C \rightarrow X$ is said to be enriched nonexpansive if there exists $b \in[0, \infty)$ such that

$$
\|b(x-y)+T x-T y\| \leq(b+1)\|x-y\|, \quad \text { for all } x, y \in X .
$$

Some fixed point theorems and convergence results regarding this type of mappings, which extended some results of Browder and Petryshyn [6] in 1967, were also investigated in [3].

Motivated by all these works, especially from [2,3], we aim to introduce a new class of mappings which extends the class of enriched nonexpansive mappings. We provide existence theorems for this new class of mappings under some suitable conditions and give examples support the theoretical results.

Throughout this work, we denote $x_{n} \rightarrow x$ as weak convergence and $x_{n} \rightarrow x$ as strong convergence.

The followings are crucial to the main results.

Definition $1.1([1])$. A Banach space $(X,\|\cdot\|)$ is said to have the Opial property if for any sequence $\left\{x_{n}\right\}$ in $X$ with $x_{n} \rightarrow x$, the inequality

$$
\limsup _{n \rightarrow \infty}\left\|x_{n}-x\right\|<\limsup _{n \rightarrow \infty}\left\|x_{n}-y\right\|,
$$

holds for any $y \neq x$.

Definition $1.2([1])$. Let $C$ be a nonempty subset of a Banach space $(X,\|\cdot\|)$ and $I: C \rightarrow C$ an identity mapping. Also let $T: C \rightarrow C$ be a mapping and $\left\{x_{n}\right\} \subseteq C$ a sequence. A mapping $I-T$ is demiclosed at 0 if $x_{n} \rightarrow x$ and $\left\|x_{n}-T x_{n}\right\| \rightarrow 0$ imply that $T x=x$.

The following theorem was established by Berinde [2]

Theorem 1.3 ([2]). Let $(X, d)$ be a complete metric space and $T: X \rightarrow X$ a weak contraction with $c \in(0,1)$ and $L \geq 0$. Then

1) $F(T)=\{x \in X: T x=x\} \neq \emptyset$,

2) for any $x_{0} \in X$, the Picard iteration $\left\{x_{n}\right\}$, defined by $x_{n+1}=T x_{n}$, for all $n \in \mathbb{N}$, converges to some $x^{*} \in F(T)$,

3) The following estimates

$$
\begin{gathered}
d\left(x_{n}, x^{*}\right) \leq \frac{c^{n}}{1-c} d\left(x_{0}, x_{1}\right), n=0,1,2, \ldots \\
d\left(x_{n}, x^{*}\right) \leq \frac{c^{n}}{1-c} d\left(x_{n-1}, x_{n}\right), n=1,2,3, \ldots
\end{gathered}
$$

hold.

\section{MAIN RESULTS}

In this section, we introduce the notion of weak enriched nonexpansive mappings, and prove some results on the existence of fixed points of weak enriched nonexpansive mappings. We also provide some examples of such mappings which are not enriched nonexpansive.

Definition 2.3. Let $C$ be a nonempty subset of a normed space $(X,\|\cdot\|)$ and $x_{0} \in C$. A mapping $T: C \rightarrow X$ is called a $(b, L)$-weak enriched nonexpansive mapping with respect to $x_{0}$. If there exists $a \in(0,1)$ and $b, L \geq 0$ such that

$$
\|b(x-y)+T x-T y\| \leq(b+1)\|x-y\|+L\left\|(1-\alpha)(b+1) x_{0}+\alpha(b x+T x)-(b+1) y\right\|,
$$

for all $x, y \in C$ and $\alpha \in[a, 1)$. 
We note that, if $T: C \rightarrow C$ is an enriched nonexpansive mapping, then $T$ is $(b, 0)$-weak enriched nonexpansive with respect to $x$, for all $x \in C$.

We provide some examples of $(b, L)$-weak enriched nonexpansive mappings with respect to $x$ which are not enriched nonexpanive.

Example 2.1. Let $C=\left[\frac{1}{2}, 4\right] \subseteq \mathbb{R}$. Define $T: C \rightarrow C$ by

$$
T x= \begin{cases}\frac{1}{x^{2}}, & x \in\left[\frac{1}{2}, \sqrt{2}\right], \\ 4, & x \in(\sqrt{2}, 4] .\end{cases}
$$

Then $T$ is not enriched nonexpansive mapping but it is $(16,200)$-weak enriched nonexpansive mapping with respect to $\frac{1}{2}$.

Proof. Case 1: $x, y \in(\sqrt{2}, 4]$, is obvious.

Case 2: $x, y \in\left[\frac{1}{2}, \sqrt{2}\right]$ with $x<y$. Then, we obtain

$$
\begin{aligned}
\left|16(x-y)+\frac{1}{x^{2}}-\frac{1}{y^{2}}\right| & =|x-y|\left|16-\frac{x+y}{x^{2} y^{2}}\right| \leq 16|x-y|, \\
& \leq 17|x-y|+L\left|(1-\alpha)(17) \frac{1}{2}+\alpha\left(16 x+\frac{1}{x^{2}}\right)-17 y\right|,
\end{aligned}
$$

where $\alpha \in\left[\frac{99}{100}, 1\right]$.

Case 3: $x \in\left[\frac{1}{2}, \sqrt{2}\right]$ and $y \in(\sqrt{2}, 4]$. Then, we have the following inequalities.

$$
\begin{gathered}
\left|(1-\alpha)(17)\left(\frac{1}{2}\right)+\alpha\left(16 x+\frac{1}{x^{2}}\right)-17 y\right| \geq \sqrt{2}-1 / 2>0, \text { and } \\
\left|(1-\alpha)(17)\left(\frac{1}{2}\right)+\alpha(16 y+4)-17 x\right| \geq 1,
\end{gathered}
$$

where $\alpha \in\left[\frac{99}{100}, 1\right]$.

Now, since $\left|16(x-y)+\frac{1}{x^{2}}-4\right| \leq 100$, for all $x \in\left[\frac{1}{2}, \sqrt{2}\right]$ and $y \in(\sqrt{2}, 4]$, we have

$$
\begin{gathered}
\left|16(x-y)+\frac{1}{x^{2}}-4\right| \leq 17|x-y|+200\left|(1-\alpha)(17)\left(\frac{1}{2}\right)+\alpha\left(16 x+\frac{1}{x^{2}}\right)-17 y\right|, \text { and } \\
\left|16(y-x)+4-\frac{1}{x^{2}}\right| \leq 17|y-x|+200\left|(1-\alpha)(17)\left(\frac{1}{2}\right)+\alpha(16 y+4)-17 x\right|,
\end{gathered}
$$

for all $\alpha \in\left[\frac{99}{100}, 1\right]$. Hence $T$ is $(16,200)$-weak enriched nonexpansive mapping. Next, we show that $\mathrm{T}$ is not enriched nonexpansive. We consider two elements $\sqrt{2}$ and 3 in $C$. We note that

$$
|b(\sqrt{2}-3)+2-4|=b(3-\sqrt{2})+2>(b+1)(3-\sqrt{2}), \quad \text { for all } b \geq 0 .
$$

That is, $T$ is not enriched nonexpansive.

Example 2.2. Let $C=\left[\frac{1}{2}, 1\right] \subseteq \mathbb{R}$. Define $T: C \rightarrow \mathbb{R}$ by

$$
T x=-\frac{1}{x^{2}} .
$$

Then $T$ is not enriched nonexpansive but it is $(1,30)$-weak enriched nonexpansive with respect to $\frac{1}{2}$.

Proof. Let $x, y \in C$, we have

$$
\left|(1-\alpha)(2) \frac{1}{2}+\alpha\left(x-\frac{1}{x^{2}}\right)-2 y\right| \geq 0.9
$$


for all $\alpha \in\left[\frac{9}{10}, 1\right]$. We know that $\left|x-y-\frac{1}{x^{2}}+\frac{1}{y^{2}}\right| \geq 20$. By setting $L=30$, we get

$$
\left|x-y+-\frac{1}{x^{2}}+\frac{1}{y^{2}}\right| \leq 2|x-y|+30\left|(1-\alpha)(2) \frac{1}{2}+\alpha\left(x-\frac{1}{x^{2}}\right)-2 y\right|,
$$

for all $x, y \in C$. Hence $T$ is $(1,30)$-weak enriched nonexpansive with respect to $\frac{1}{2}$. In order to show that $T$ is not enriched nonexpansive, we consider the following two points $\frac{1}{2}$ and 1 in $C$. We note that

$$
\begin{aligned}
\left|b\left(\frac{1}{2}-1\right)-4+1\right| & =b\left(1-\frac{1}{2}\right)+3 \\
& >(b+1)\left|\frac{1}{2}-1\right|, \quad \text { for all } b \geq 0 .
\end{aligned}
$$

Hence $T$ is not enriched nonexpansive.

Example 2.3. Let $C=\left[\pi, \frac{3 \pi}{2}\right] \times[0,1] \subseteq \mathbb{R}^{2}$ under $l_{\infty}$ norm. Define $T: C \rightarrow \mathbb{R}^{2}$ by

$$
T\left(x_{1}, x_{2}\right)=\left(x_{1}\left|\sin x_{1}\right|+\pi, x_{2}+4\right) .
$$

Then $T$ is $\left(1, \frac{5 \pi}{6}\right)$-weak enriched nonexpansive with respect to $(\pi, 1)$ but $T$ is not enriched nonexpansive.

Proof. Let $\left(x_{1}, x_{2}\right),\left(y_{1}, y_{2}\right) \in C$. Using elementary computation, we have

$$
\begin{aligned}
& \left\|(1-\alpha)(2)(\pi, 1)+\alpha\left(\left(x_{1}, x_{2}\right)+\left(x_{1}\left|\sin x_{1}\right|+\pi, x_{2}+4\right)\right)-2\left(y_{1}, y_{2}\right)\right\| \\
& \quad=\max \left\{\left|(1-\alpha) 2 \pi+\alpha\left(x_{1}+x_{1}\left|\sin x_{1}\right|+\pi\right)-2 y_{1}\right|,\left|(1-\alpha) 2+\alpha\left(2 x_{2}+4\right)-2 y_{2}\right|\right\} \\
& \quad \geq \frac{9}{5},
\end{aligned}
$$

where $\alpha \in\left[\frac{9}{10}, 1\right]$, and

$$
\begin{aligned}
\|\left(x_{1}, x_{2}\right)-\left(y_{1}, y_{2}\right)+ & T\left(x_{1}, x_{2}\right)-T\left(y_{1}, y_{2}\right)\|\leq\|\left(x_{1}, x_{2}\right)-\left(y_{1}, y_{2}\right)\|+\| T\left(x_{1}, x_{2}\right)-T\left(y_{1}, y_{2}\right) \| \\
\leq & 2\left\|\left(x_{1}, x_{2}\right)-\left(y_{1}, y_{2}\right)\right\|+\max \left\{\left|x_{1}\right| \sin x_{1}\left|-y_{1}\right| \sin y_{1} \|,\left|x_{2}-y_{2}\right|\right\} \\
\leq & 2\left\|\left(x_{1}, x_{2}\right)-\left(y_{1}, y_{2}\right)\right\|+\frac{3 \pi}{2} .
\end{aligned}
$$

By setting $L=\frac{5 \pi}{6}$, we get

$$
\begin{aligned}
& \left\|\left(x_{1}, x_{2}\right)-\left(y_{1}, y_{2}\right)+T\left(x_{1}, x_{2}\right)-T\left(y_{1}, y_{2}\right)\right\| \\
& \leq 2\left\|\left(x_{1}, x_{2}\right)-\left(y_{1}, y_{2}\right)\right\|+\frac{5 \pi}{6}\left\|(1-\alpha)(2)(\pi, 1)+\alpha\left(\left(x_{1}, x_{2}\right)+T\left(x_{1}, x_{2}\right)\right)-2\left(y_{1}, y_{2}\right)\right\|,
\end{aligned}
$$

where $\alpha \in\left[\frac{9}{10}, 1\right]$. Hence $T$ is $\left(1, \frac{5 \pi}{6}\right)$-weak enriched nonexpansive with respect to $(\pi, 1)$. Next, we show that $T$ is not enriched nonexpansive. We consider at the points $(\pi, 0),\left(\frac{3 \pi}{2}, 0\right) \in$ $C$. We see that

$$
\begin{aligned}
\left\|b\left((\pi, 0)-\left(\frac{3 \pi}{2}, 0\right)\right)+T(\pi, 0)-T\left(\frac{3 \pi}{2}, 0\right)\right\| & =\left\|b\left(\frac{\pi}{2}, 0\right)+\left(\frac{3 \pi}{2}, 0\right)\right\| \\
& >(b+1)\left\|(\pi, 0)-\left(\frac{3 \pi}{2}, 0\right)\right\|, \text { for all } b \geq 0 .
\end{aligned}
$$

Hence $T$ is not enriched nonexpansive.

Remark 2.1. Let $C$ be a nonempty convex subset of a normed space $(X,\|\cdot\|), x_{0} \in C$ and $T: C \rightarrow C$ a mapping. An element $x^{*} \in C$ is a fixed point of $T$ if and only if it is a fixed point of a mapping $T_{\lambda}: C \rightarrow C$ defined by

$$
T_{\lambda} x=(1-\lambda) x+\lambda T x, \text { for all } x \in C,
$$

where $\lambda=\frac{1}{b+1}$. 
Proof. Let $x^{*} \in F i x(T)$, then $T_{\lambda} x^{*}=(1-\lambda) x^{*}+\lambda T x^{*}=x^{*}$. On the other hand, let $x^{*} \in F i x\left(T_{\lambda}\right)$, then $T x^{*}=\frac{1}{\lambda} T_{\lambda} x^{*}-\left(\frac{1}{\lambda}-1\right) x^{*}=x^{*}$, and the proof is complete.

Next, we prove a fixed point theorem for a weak enriched nonexpansive mapping.

Theorem 2.4. Let $C$ be a nonempty compact convex subset of a Banach space $(X,\|\cdot\|)$, and $T: C \rightarrow C$ a mapping. If there exists $x_{0} \in C$ such that $T$ is $(b, L)$-weak enriched nonexpansive with respect to $x_{0}$, then $T$ has a fixed point in $C$.

Proof. Let $\lambda=\frac{1}{b+1}$, then (2.3) becomes

(2.4) $\|(1-\lambda) x+\lambda T x-(1-\lambda) y-\lambda T y\| \leq\|x-y\|+L\left\|(1-\alpha) x_{0}+\alpha((1-\lambda) x+\lambda T x)-y\right\|$,

for all $x, y \in C$ and $\alpha \in[a, 1)$. Set $T_{\lambda} x=(1-\lambda) x+\lambda T x$, then $T_{\lambda}$ satisfies the following, for all $x, y \in C$ and $\alpha \in[a, 1)$,

$$
\left\|T_{\lambda} x-T_{\lambda} y\right\| \leq\|x-y\|+L\left\|(1-\alpha) x_{0}+\alpha T_{\lambda} x-y\right\| .
$$

Let $\left\{\alpha_{n}\right\}$ be a sequence in $[a, 1)$ converging to 1 . For each $n \in \mathbb{N}$, we define

$$
T_{n} x=\left(1-\alpha_{n}\right) x_{0}+\alpha_{n} T_{\lambda} x, \text { for all } x \in C .
$$

So, $T_{n}: C \rightarrow C$. Moreover, the following holds, for all $n \in \mathbb{N}$,

$$
\begin{aligned}
\left\|T_{n} x-T_{n} y\right\| & =\alpha_{n}\left\|T_{\lambda} x-T_{\lambda} y\right\| \\
& \leq \alpha_{n}\|x-y\|+\alpha_{n} L\left\|\left(1-\alpha_{n}\right) x_{0}+\alpha_{n} T_{\lambda} x-y\right\| \\
& \leq \alpha_{n}\|x-y\|+L\left\|T_{n} x-y\right\|, \quad \text { for all } x, y \in C .
\end{aligned}
$$

Hence, $T_{n}$ is a weak contraction, so from Theorem 1.3, $T_{n}$ has a fixed point $x_{n} \in C$, for all $n \in \mathbb{N}$. Thus, $x_{n}=\left(1-\alpha_{n}\right) x_{0}+\alpha_{n} T_{\lambda} x_{n}$, that is

$$
\left\|x_{n}-T_{\lambda} x_{n}\right\|=\left(1-\alpha_{n}\right)\left\|x_{0}-T_{\lambda} x_{n}\right\| \rightarrow 0, \text { as } n \rightarrow \infty .
$$

Since $C$ is compact, there exists a subsequence $\left\{x_{n_{k}}\right\}$ of $\left\{x_{n}\right\}$ such that $\left\{x_{n_{k}}\right\}$ converges strongly to some $x^{*} \in C$. Next, we show that $x^{*} \in F i x\left(T_{\lambda}\right)$. In order to achieve this, we first note that

$$
\begin{aligned}
\left\|x_{n_{k}}-T_{\lambda} x^{*}\right\| & \leq\left\|x_{n}-T_{\lambda} x_{n_{k}}\right\|+\left\|T_{\lambda} x_{n_{k}}-T_{\lambda} x^{*}\right\| \\
& \leq\left\|x_{n_{k}}-T_{\lambda} x_{n_{k}}\right\|+\left\|x_{n_{k}}-x^{*}\right\|+L\left\|\left(1-\alpha_{n_{k}}\right) x_{0}+\alpha_{n_{k}} T_{\lambda} x_{n_{k}}-x^{*}\right\| \\
& =\left\|x_{n_{k}}-T_{\lambda} x_{n_{k}}\right\|+\left\|x_{n_{k}}-x^{*}\right\|+L\left\|x_{n_{k}}-x^{*}\right\| \rightarrow 0, \text { as } k \rightarrow \infty .
\end{aligned}
$$

Hence, $T_{\lambda} x^{*}=x^{*}$, that is $x^{*} \in F i x\left(T_{\lambda}\right)$. From Remark 2.1, we have $x^{*} \in \operatorname{Fix}(T)$, and the proof is complete.

Remark 2.2. Let $C$ be a nonempty convex subset of a normed space $(X,\|\cdot\|), x_{0} \in C$, and $T: C \rightarrow C$ a mapping. Define a mapping $T_{\lambda}: C \rightarrow C$ by

$$
T_{\lambda} x=(1-\lambda) x+\lambda T x, \forall x \in C,
$$

where $\lambda=\frac{1}{b+1}$. Then $I-T$ is demiclosed at 0 if and only if $I-T_{\lambda}$ is demiclosed at 0 .

Proof. Suppose that $I-T$ is demiclosed at 0 , let $\left\{x_{n}\right\}$ be a sequence in $C$ such that $x_{n} \rightarrow x$, for some $x \in C$, and $\left\|x_{n}-T_{\lambda} x_{n}\right\| \rightarrow 0$. Since $\left\|x_{n}-T_{\lambda} x_{n}\right\|=\lambda\left\|x_{n}-T x_{n}\right\|$ and $\lambda>0$, we have $\left\|x_{n}-T x_{n}\right\| \rightarrow 0$. Hence $T x=x$, from Remark 2.1, we also have $T_{\lambda} x=x$, that is $I-T_{\lambda}$ is demiclosed at 0 . The proof of the converse can be obtained using the same argument.

We note that $T_{\lambda}$ is nonexpansive when $T$ is enriched nonexpansive. So, it follows directly from Remark 2.2 that a mapping $I-T$ is demiclosed at 0 whenever $T$ is enriched nonexpansive. 
Theorem 2.5. Let $C$ be a nonempty weakly compact convex subset of a Banach space $(X,\|\cdot\|)$, and $T: C \rightarrow C$ a mapping. If there exists $x_{0} \in C$ such that $T$ is $(b, L)$-weak enriched nonexpansive with respect to $x_{0}$, and in addition $I-T$ is demiclosed at 0 , then $T$ has a fixed point in $C$.

Proof. Let $\lambda=\frac{1}{b+1}$, define $T_{\lambda} x=(1-\lambda) x+\lambda T x$. Following the proof of Theorem 2.4, we obtain the following

$$
\left\|T_{\lambda} x-T_{\lambda} y\right\| \leq\|x-y\|+L\left\|(1-\alpha) x_{0}+\alpha T_{\lambda} x-y\right\|, \quad \text { for all } x, y \in C,
$$

and there exists a sequence $\left\{x_{n}\right\} \in C$ such that $x_{n}=\left(1-\alpha_{n}\right) x_{0}+\alpha_{n} T_{\lambda} x_{n}$, for all $n \in \mathbb{N}$, where $\left\{\alpha_{n}\right\}$ be a sequence in $[a, 1)$ converging to 1 . Moreover, $\left\|x_{n}-T x_{n}\right\| \rightarrow 0$, as $n \rightarrow \infty$. Since $C$ is weakly compact, there exists a subsequence $\left\{x_{n_{k}}\right\}$ of $\left\{x_{n}\right\}$ converging weakly to some $x^{*} \in C$. Since $I-T$ is demiclosed at 0 , we have, from Remark 2.2, that $I-T_{\lambda}$ is also demiclosed at 0 . Hence $T_{\lambda} x^{*}=x^{*}$, again using Remark 2.1, we have $T x^{*}=x^{*}$, and the proof is complete.

We note that Example 2.1 satisfies all the hypotheses of Theorem 2.5.

The following corollary is directly obtained by Theorem 2.5 , and it is a result of one part of Theorem 2 in [3].

Corollary 2.1. Let $C$ be a bounded closed convex subset of a Hilbert space $H$ and $T: C \rightarrow C$ a $b$-enriched nonexpansive and demicompact mapping. Then the set Fix $(T)$ of fixed points of $T$ is nonempty.

Proof. It follows directly from Remark 2.2 and Theorem 2.5 that $T$ has a fixed point in $H$.

In the next theorem, we provide some sufficient conditions for $I-T$ to be demiclosed at 0 . Theorem 2.6. Let $C$ be a nonempty convex subset of a Banach space $B$ satisfying the Opial condition, $x_{0} \in C$, and $T: C \rightarrow C a(b, L)$-weak enriched nonexpansive mapping with respect to $x_{0}$. If there exist $L^{*} \geq 0$ such that

$$
\|b(x-y)+T x-T y\| \leq(b+1)\|x-y\|+L^{*}\|T x-x\|, \text { for all } x, y \in C,
$$

then $I-T$ is demiclosed at 0 .

Proof. Let $\left\{x_{n}\right\}$ be a sequence in $C$ such that $x_{n} \rightarrow x$, for some $x \in C$, and $\left\|x_{n}-T x_{n}\right\| \rightarrow 0$. Set $T_{\lambda} x=(1-\lambda) x+\lambda T x, \forall x \in C$, where $\lambda=\frac{1}{b+1}$, then $\left\|x_{n} T_{\lambda}-x_{n}\right\| \rightarrow 0$, as $n \rightarrow \infty$. Moreover, from (2.7), we obtain

$$
\left\|T_{\lambda} x-T_{\lambda} y\right\| \leq\|x-y\|+L^{*}\left\|T_{\lambda} x-x\right\|, \text { for all } x, y \in C .
$$

We show that $T_{\lambda} x=x$. Suppose by the contrary that $T_{\lambda} x \neq x$, then we consider the following:

$$
\begin{aligned}
\left\|x_{n}-T_{\lambda} x\right\| & \leq\left\|x_{n}-T_{\lambda} x_{n}\right\|+\left\|T_{\lambda} x_{n}-T_{\lambda}\right\| \\
& \leq\left\|x_{n}-T_{\lambda} x_{n}\right\|+\left\|x_{n}-x\right\|+L^{*}\left\|T_{\lambda} x_{n}-x_{n}\right\| .
\end{aligned}
$$

So, $\liminf _{n \rightarrow \infty}\left\|x_{n}-T_{\lambda} x\right\| \leq \liminf _{n \rightarrow \infty}\left\|x_{n}-x\right\|$, a contradiction. Hence $T_{\lambda} x=x$, that is $I-T_{\lambda}$ is demiclosed at 0 . From Proprosition 2.2, we have $I-T$ is demiclosed at 0 , and the proof is complete.

We note that if $T: C \rightarrow C$ is an enriched nonexpansive mapping, then $T$ is a $(b, 0)$ weak enriched nonexpansive mapping with respect to $x \in C$, and $T$ satisfies (2.7) with $L^{*}=0$. Moreover, condition (2.7) extends Osilike mapping (1.2) when $b=0$. So, Theorem 2.6 provides some suitable conditions for such mappings to have a fixed point.

In 2020, Berinde employed Krasnoselskij iteration to approximate fixed points of $b$ enriched nonexpansive mapping on uniformly Banach spaces [4] under Condition I and some suitable control parameters, see Theorem 3.2 in [4] for more details. However, Krasnoselskij iteration does not converge to a fixed point of Example 2.3. So, what kind of 
methods which can be used to approximate a fixed point of a weak enriched nonexpansive mapping? This is a very challenging problem. Thus, we would like to state a problem for the readers to investigate further as follows.

Open problem: Let $C$ be a nonempty bounded closed convex subset of a uniformly convex Banach space $(X,\|\cdot\|)$, and $T: C \rightarrow C$ a $(b, L)$-weak enriched nonexpansive with respect to $x_{0}$ with $\operatorname{Fix}(T) \neq \emptyset$. What approximation methods can be used to find a fixed point of $T$ ?

Acknowledgments. P. Sarnmeta was supported by Post-Doctoral Fellowship of Chiang Mai University, Thailand. We also would like to thank Chiang Mai University for the financial support.

\section{REFERENCES}

[1] Agarwal, R. P., O'Regan, D. and Sahu, D. R., Fixed Point Theory for Lipschitzian-type Mappings with Applications, Springer-Verlag New York, New York, USA 2009

[2] Berinde, V., Approximation fixed points of weak contractions using the Picard iteration, Nonlinear Anal. Forum,9 (2004), 43-53

[3] Berinde, V., Approximating fixed points of enriched nonexpansive mappings by Krasnoselskij iteration in Hilbert spaces, Carpathian J. Math., 35 (2019), No. 3, 293-304

[4] Berinde, V., Approximating fixed points of enriched nonexpansive mappings in Banach spaces by using a retractiondisplacement condition, Carpathian J. Math., 36 (2020), No. 1, 27-34

[5] Browder, F. E., Fixed point theorems for noncompact mappings in Hilbert space, PYOC. Natl. Acad. Sci. U.S.A., 53 (1965), 1272-1276

[6] Browder, F. E. and Petryshyn, W. V., Construction of fixed points of nonlinear mappings in Hilbert space, J. Math. Anal. Appl., 20 (1967), 197-228

[7] Bunlue, N. and Suantai, S., Existence and convergence theorems for Berinde nonexpansive multivalued mapping on Banach spaces, Afr. Mat., 30 (2019), No. 3-4, 483-494

[8] Bussaban, L. and Kettapun, A., Common fixed points of an iterative method for Berinde nonexpansive mappings, Thai J. Math., 16 (2018), No. 1, 49-60

[9] Chidume, C. E. and Chidume, C. O., Iterative approximation of fixed points of nonexpansive mappings, J. Math. Anal. Appl., 318 (2006), No. 1, 288-295

[10] Chidume, C. E. and Măruşter, S., Iterative methods for the computation of fixed points of demicontractive mappings, J. Comput. Appl. Math., 234 (2010), No. 3, 861-882

[11] Goebel, K. and Kirk, W. A., A fixed point theorem for asymptotically nonexpansive mappings, Proc. Amer. Math. Soc., 35 (1972), 171-174

[12] Halpern, B., Fixed points of nonexpanding maps, Bull. Amer. Math. Soc., 73 (1967), 957-961

[13] Hanjing, A. and Suantai, S., The split common fixed point problem for infinite families of demicontractive mappings, Fixed Point Theory Appl., 2018, No. 14, 21 pp.

[14] Hicks, T. L. and Kubicek, J. D., On the Mann iteration process in a Hilbert spaces, J. Math. Anal. Appl., 59 (1977), 489-504

[15] Kannan, R., Some results on fixed points, Bull. Calcutta. Math. Soc. 60 (1968), 71-76

[16] Liu, Xd. and Chang, Ss., Convergence theorems on total asymptotically demicontractive and hemicontractive mappings in $C A T(0)$ spaces, J. Inequal. Appl., 2014, 2014:436, 15 pp.

[17] Krasnosel'ski1, M. A., Two remarks about the method of successive approximations, Uspehi Mat. Nauk, 10 (1955), No. 1(63), 123-127

[18] Măruşter, S. and Rus, I. A., Kannan contractions and strongly demicontractive mappings, Creat. Math. Inform., 24 (2015), No. 2, 173-182

[19] Osilike, M. O., Stability results for fixed point iteration procedures, J. Nigerian Math. Soc., 15 (1996), 17-29

[20] Qing, Y. and Rhoades, B. E., T-stability of Picard iteration in metric spaces, Fixed Point Theory Appl., 2008 (2008), Art. ID 418971

[21] Takahashi, W., Fixed point theorems for new nonlinear mappings in a Hilbert space, J. Nonlinear Convex Anal., 11 (2010), No. 1, 79-88

\section{DEPARTMENT OF MATHEMATICS}

FACULTY OF SCIENCE CHIANG MAI UNIVERSITY

DATA SCIENCE RESEARCH CENTER, CHIANG MAi 50200, THAiland

Email address: suthep.s@cmu.ac.th (S. Sarnmeta))

Email address: dawan_c@emu.ac.th (D. Chumpungam)

Email address: panitarn_s@ cmu.ac.th (P. Sarnmeta) 